\title{
Sensitivity Analysis for Interval-valued Fully Fuzzy Linear Programming Problems
}

\author{
Neha Bhatia ${ }^{* 1}$, Amit Kumar ${ }^{2}$ \\ ${ }^{1,2}$ School of Mathematics and Computer Applications \\ Thapar University, Patiala-147004, India \\ *renam.thaparian@gmail.com \\ 1 School of Mathematics and Computer Applications \\ Thapar University, Patiala-147004, India
}

\begin{abstract}
In previous studies, it is pointed out that in several situations it is better to use interval-valued fuzzy numbers instead of triangular or trapezoidal fuzzy numbers. But till now, there is no method that deals with the sensitivity analysis of such linear programming problems in which all the parameters are represented by interval-valued fuzzy numbers. In this paper, a new method is proposed for the sensitivity analysis. Finally, the proposed method is illustrated using a numerical example.
\end{abstract}

Keywords: Interval-valued fuzzy numbers, interval-valued fully fuzzy linear programming problems, sensitivity analysis, ranking function.

\section{Introduction}

The sensitivity analysis is a wellexplored area in classical linear programming. The sensitivity analysis is a basic tool for studying perturbations in optimization problems. There is considerable research on sensitivity analysis for some operations research and management science models such as linear programming and investment analysis.

In the real word, there are many problems which have linear programming models and sometimes it is necessary to formulate these models with parameters of uncertainty. Many numbers from these problems are linear programming problems with fuzzy variables. In most practical applications of mathematical programming the possible values of the parameters required in the modeling of the problem are provided either by a decision maker subjectively or by a statistical inference from the past data because there is some uncertainty. In order to reflect this uncertainty, the model of the problem is often constructed with fuzzy data [1].

The concept of fuzzy mathematical programming on a general level was first proposed by Tanaka et al. [2] in the framework of the fuzzy decision of Bellman and Zadeh [3]. The first formulation of fuzzy linear programming was proposed by

Zimmermann [4]. Afterwards, many authors have considered several kinds of fuzzy linear programming problems and have proposed several approaches for solving these problems. Maleki et al. [5] proposed a new method for solving the fuzzy number linear programming problem and used its solution to obtain the fuzzy solution of the fuzzy variable linear programming problem.

Chiang [6] used statistical data and statistical confidence interval to derive $(1-\alpha)$ fuzzy numbers, $(0<\alpha<1)$ and got a fuzzy linear programming problem. Chiang used two statistical confidence intervals to derive $(1-\beta, 1-\alpha)$ intervalvalued fuzzy numbers, $0<\alpha<\beta<1$ and got another fuzzy linear programming problem.

Mahdavi-Amiri and Nasseri [7] extended the concepts of duality in fuzzy number linear programming problems. Ganesan and Veeramani [8] introduced a new method for solving a kind of linear programming problem involving symmetric trapezoidal fuzzy numbers without converting them to crisp linear programming problems.

Su [9] proposed a method for fuzzy linear programming based on interval-valued fuzzy 
numbers and ranking. Mahdavi-Amiri and Nasseri [10] used a certain linear ranking function to define the duality of fuzzy number linear programming problems and proposed the dual simplex algorithm for solving fuzzy variables linear programming problems. Moreover, Ebrahimnejad and Nasseri [11] used the complementary slackness property to solve fuzzy number linear programming problems and fuzzy variables linear programming problems without the need of a simplex table.

Hosseinzadeh Lotfi et al. [12] proposed a method to obtain the approximate solution of fully fuzzy linear programming problems. Ebrahimnejad and Nasseri [13] introduced a new method called the bounded dual simplex method for bounded fuzzy number linear programming problems in which some or all the variables are restricted to remain within fuzzy lower and fuzzy upper bounds. Ebrahimnejad et al. [14] developed a method for solving such fuzzy linear programming problems in which some or all the fuzzy decision variables were bounded. Ebrahimnejad et al. [15] proposed a method namely primal-dual simplex algorithm to obtain a fuzzy solution of fuzzy variables linear programming problems. Nasseri and Ebrahimnejad [16] applied a fuzzy primal simplex method to solve flexible linear programming problems directly without solving any auxiliary problem.

Kheirfam and Hasani [17] studied the basis of invariance sensitivity analysis for fuzzy linear programming problems. Kumar and Bhatia [18] proposed a method for the sensitivity analysis for fuzzy linear programming problems with fuzzy variables by converting them into a crisp linear programming problem. Kumar and Bhatia [19] proposed a new method that deals with the sensitivity analysis of such FLP problems in which only the elements of the coefficient matrix of constraints are represented by real numbers and other parameters are represented by trapezoidal fuzzy numbers to overcome this limitation of existing method [17].

Ebrahimnejad [20] generalized the concept of sensitivity analysis in fuzzy number linear programming problems by applying fuzzy simplex algorithms and using the general linear ranking function on fuzzy numbers. Kumar and Bhatia [21] proposed a method to overcome the limitation of the method proposed by Ebrahimnejad [20].
Nasseri and Ebrahimnejad [22] proposed a method for sensitivity analysis on linear programming problem with trapezoidal fuzzy variables. Bhatia and Kumar [23] proposed a method to deal with the sensitivity analysis of such fuzzy linear programming problems in which all the parameters are represented by $L R$ flat fuzzy numbers. HatamiMarbini and Tavana [24] proposed a 'general' and 'interactive' method for solving linear programming problems with fuzzy parameters.

In previous studies it was pointed out that the values of the membership of an element, in a fuzzy set, are represented by a real number in $[0,1]$ but a specialist is always uncertain about the values of the membership. Hence, it is better to represent the values of the membership of an element in a set by intervals of possible real numbers instead of real numbers. In this paper, a new method that deals with the sensitivity analysis of such linear programming problems in which all the parameters are represented by interval-valued fuzzy numbers is proposed. Finally, the proposed method is illustrated using a numerical example.

This paper is organized as follows: In section 2, some basic definitions and arithmetic operations are presented. In section 3 , a new method, is proposed to deal with the sensitivity analysis of interval-valued fully fuzzy linear programming problems. In section 4, the proposed method is illustrated with the help of a numerical example. Finally, in section the conclusions are discussed.

\section{Preliminaries}

In this section some basic definitions, the arithmetic operations and the comparison of interval-valued fuzzy numbers, are presented.

\subsection{Basic definitions}

In this section, some basic definitions are presented [25].

Definition 2.1: If the membership function of the fuzzy set $\tilde{A}=(a, b, c)$ on $R=(-\infty, \infty)$ is

$$
\mu_{\tilde{A}}(x)= \begin{cases}1, & x=a \\ 0, & x \neq a\end{cases}
$$


then $\tilde{A}$ is called a fuzzy point.

Definition 2.2: If the membership function of the fuzzy set $\tilde{A}$ on $R$ is

$$
\mu_{\tilde{A}}(x)=\left\{\begin{array}{cl}
\frac{\lambda(x-a)}{(b-a)}, & a<x \leq b \\
\frac{\lambda(c-x)}{(c-b)}, & b \leq x<c \\
0, & \text { otherwise }
\end{array}\right.
$$

$a<b<c$, then $\tilde{A}$ is called $\lambda$ fuzzy number, $0<\lambda \leq 1$ and is denoted as $\tilde{A}=(a, b, c ; \lambda)$.

Let $F_{N}(\lambda)$ be the family of all $\lambda$ fuzzy numbers, i.e.

$$
\begin{aligned}
& F_{N}(\lambda)=\{(a, b, c ; \lambda): \forall a<b<c, a, b, c \in R\} \\
& 0<\lambda \leq 1 .
\end{aligned}
$$

Definition 2.3: An interval-valued fuzzy set $\tilde{A}$ on $R$ is given by

$$
\tilde{A}=\left\{\left(x,\left[\mu_{\tilde{A}^{L}}(x), \mu_{\tilde{A}^{U}}(x)\right]\right): x \in R\right\},
$$

where $\quad \mu_{\tilde{A}^{L}}(x), \mu_{\tilde{A}^{U}}(x) \in[0,1] \quad$ and $\quad \mu_{\tilde{A}^{L}}(x) \leq \mu_{\tilde{A}^{U}}(x)$ $\forall x \in R$ and are denoted as $\tilde{A}=\left[\tilde{A}^{L}, \tilde{A}^{U}\right]$.

This means that the grade of membership of $x$ belongs to the interval $\left[\mu_{\tilde{A}^{L}}(x), \mu_{\tilde{A}^{u}}(x)\right]$, the least grade of membership at $x$ is $\mu_{\tilde{A}^{L}}(x)$ and the greatest grade of the membership at $x$ is $\mu_{\tilde{A}^{U}}(x)$.

The interval-valued fuzzy sets were first used by Gorzafczany [26] and have been applied to the fields of approximate inference, to the signal transmission and to the controller, etc.
Let

$$
\mu_{\tilde{A}^{L}}(x)=\left\{\begin{array}{cl}
\frac{\lambda(x-a)}{(b-a)}, & a<x \leq b \\
\frac{\lambda(c-x)}{(c-b)}, & b \leq x<c \\
0, & \text { otherwise }
\end{array}\right.
$$

Then $\tilde{A}^{L}=(a, b, c ; \lambda)$.

Let

$\mu_{\tilde{A}^{U}}(x)=\left\{\begin{array}{cl}\frac{\rho(x-p)}{(b-p)}, & p<x \leq b \\ \frac{\rho(r-x)}{(r-b)}, & b \leq x<r \\ 0, & \text { otherwise }\end{array}\right.$

Then $\tilde{A}^{U}=(p, b, r ; \rho)$. Here $0<\lambda \leq \rho \leq 1$ and $p<a<b<c<r$. The interval-valued fuzzy set $\tilde{A}=[(a, b, c ; \lambda),(p, b, r ; \rho)]$. is called $(\lambda, \rho)$ intervalvalued fuzzy number.

Remark 2.1 When $\lambda=0, a=p, c=r, \quad \tilde{A}=\left[\tilde{A}^{L}, \tilde{A}^{U}\right]$ will be reduced to $\rho$ fuzzy number $\tilde{A}^{U}=(p, b, r ; \rho)$.

\subsection{Arithmetic operations [25]}

The arithmetic operations between two $(\lambda, \rho)$ interval-valued fuzzy numbers

$\tilde{A}=\left[\left(a_{1}, b_{1}, c_{1} ; \lambda\right),\left(a_{2}, b_{1}, c_{2} ; \rho\right)\right]$ and

$\left.\tilde{B}=\left[\left(p_{1}, q_{1}, r_{1} ; \lambda\right)\right],\left(p_{2}, q_{1}, r_{2} ; \rho\right)\right]$ are defined as follows:

$\tilde{A} \oplus \tilde{B}=\left[\left(a_{1}+p_{1}, b_{1}+q_{1}, c_{1}+r_{1} ; \lambda\right),\left(a_{2}+p_{2}\right.\right.$,

$\left.\left.b_{1}+q_{1}, c_{2}+r_{2} ; \rho\right)\right]$ 


$$
\tilde{A} \Theta \tilde{B}=\left[\left(a_{1}-r_{1}, b_{1}-q_{1}, c_{1}-p_{1} ; \lambda\right),\right.
$$$$
\left.\left(a_{2}-r_{2}, b_{1}-q_{1}, c_{2}-p_{2} ; \rho\right)\right]
$$

If $\tilde{A}$ and $\tilde{B}$ are two non-negative interval valued fuzzy numbers then

$$
\tilde{A} \otimes \tilde{B}=\left[\left(a_{1} p_{1}, b_{1} q_{1}, c_{1} r_{1} ; \lambda\right),\left(a_{2} p_{2}, b_{1} q_{1}, c_{2} r_{2} ; \rho\right)\right.
$$

If $k>0, k \tilde{A}=\left[\left(k a_{1}, k b_{1}, k c_{1} ; \lambda\right),\left(k a_{2}, k b_{1}, k c_{2} ; \rho\right)\right]$

If $k<0, k \tilde{A}=\left[\left(k c_{1}, k b_{1}, k a_{1} ; \lambda\right),\left(k c_{2}, k b_{1}, k a_{2} ; \rho\right)\right]$

\subsection{Comparison of interval-valued fuzzy numbers [25]}

Let $\quad \tilde{A}=\left[\left(a_{1}, b_{1}, c_{1} ; \lambda\right),\left(a_{2}, b_{1}, c_{2} ; \rho\right)\right]$ and

$\left.\tilde{B}=\left[\left(p_{1}, q_{1}, r_{1} ; \lambda\right)\right],\left(p_{2}, q_{1}, r_{2} ; \rho\right)\right]$ are two interval-valued fuzzy numbers, then

$$
\begin{gathered}
\tilde{A}>_{\Re} \tilde{B} \text { if } \mathfrak{R}(\tilde{A})>\mathfrak{R}(\tilde{B}) \\
\tilde{A}=_{\Re} \tilde{B} \text { if } \mathfrak{R}(\tilde{A})=\mathfrak{R}(\tilde{B}) \\
\tilde{A}<_{\mathfrak{R}} \tilde{B} \text { if } \mathfrak{R}(\tilde{A})<\mathfrak{R}(\tilde{B})
\end{gathered}
$$

where, $\mathfrak{R}(\tilde{A})=\frac{1}{8}\left[\begin{array}{l}6 b_{1}+a_{1}+c_{1}+4 a_{2}+4 c_{2} \\ +3\left(2 b_{1}-a_{2}-c_{2}\right) \frac{\lambda}{\rho}\end{array}\right]$

and $\Re(\tilde{B})=\frac{1}{8}\left[\begin{array}{l}6 q_{1}+p_{1}+r_{1}+4 p_{2}+4 r_{2} \\ +3\left(2 q_{1}-p_{2}-r_{2}\right) \frac{\lambda}{\rho}\end{array}\right]$

2.4 Interval-valued fully fuzzy linear programming problem

Any linear programming problem in which all the parameters are represented by interval-valued fuzzy numbers can be represented by $\left(\mathrm{P}_{1}\right)$ :
$\operatorname{Max}\left(\right.$ or Min) $\quad\left(\tilde{C}^{T} \otimes \tilde{X}\right)$

Subject to

$$
\tilde{A} \otimes \tilde{X} \leq_{\Re} \text { or }=_{\Re} \text { or } \geq_{\Re} \tilde{b}
$$

$\tilde{X}=\left[\tilde{x}_{j}\right]_{n \times 1} ; \tilde{x}_{j}$ are non-negative interval-valued fuzzy number

$$
\begin{aligned}
& \text { and } \tilde{X}=\left[\tilde{x}_{j}\right]_{n \times 1}, \tilde{b}=\left[\tilde{b}_{k}\right]_{m \times 1}, \tilde{A}=\left[\tilde{a}_{k j}\right]_{m \times n}, \tilde{C}^{T}= \\
& {\left[\tilde{c}_{j}\right]_{1 \times n}, \tilde{x}_{j}=\left[\left(m_{j}-\beta_{j 2}, m_{j}, m_{j}+\beta_{j 3} ; .9\right),\left(m_{j}-\right.\right.} \\
& \left.\left.\beta_{j 1}, m_{j}, m_{j}+\beta_{j 4} ; 1\right)\right], \tilde{c}_{j}=\left[\left(c_{j}-\delta_{j 2}, c_{j}, c_{j}+\delta_{j 3} ;\right.\right. \\
& \left.\lambda),\left(c_{j}-\delta_{j 1}, c_{j}, c_{j}+\delta_{j 4} ; 1\right)\right], \tilde{a}_{k j}=\left[\left(a_{k j}-\delta_{k j 2}, a_{k j},\right.\right. \\
& \left.\left.a_{k j}+\delta_{k j 3} ; \lambda\right),\left(a_{k j}-\delta_{k j 1}, a_{k j}, a_{k j}+\delta_{k j 4} ; 1\right)\right], \tilde{b}_{k}=\left[\left(b_{k}\right.\right. \\
& \left.\left.-\alpha_{k 2}, b_{k}, b_{k}+\alpha_{k 3} ; \lambda\right),\left(b_{k}-\alpha_{k 1}, b_{k}, b_{k}+\alpha_{k 4} ; 1\right)\right], 0 \\
& <\delta_{j 2}<\delta_{j 1}<c_{j}, 0<\delta_{j 3}<\delta_{j 4}, 0<\delta_{k j 2}<\delta_{k j 1}<a_{k j}, 0 \\
& <\delta_{k j 3}<\delta_{k j 4}, 0<\alpha_{k 2}<\alpha_{j 1}<b_{k}, 0<\alpha_{k 3}<\alpha_{k 4} ; j=1, \\
& 2, \ldots, n ; 0<\lambda<1 ; k=1,2, \ldots, m .
\end{aligned}
$$

\section{Proposed method}

There are few methods [17, 18, 19, 20, 21, 22, 23] that deal with the sensitivity analysis of such linear programming problems in which some or all the parameters are represented by triangular or trapezoidal fuzzy numbers. In previous works, it is pointed out that in several situations it is better to use interval-valued fuzzy numbers instead of triangular or trapezoidal fuzzy numbers, thus several authors have used interval-valued fuzzy numbers $[6,9,25]$ in different types of problems. But, as far as we know, there is no method in previous works that deals with the sensitivity analysis of such linear programming problems in which all the parameters are represented by interval-valued fuzzy numbers.

In this section, a new method is proposed for the same kind of problems. The steps of the proposed method are as follows:

Step 1 Since in the existing method [6; 9] the optimal solution of the interval-valued fuzzy linear programming problem is obtained first by converting the problem into a crisp linear programming one and solving it. Here, we too convert the chosen fuzzy linear programming 
problem first $\left(\mathrm{P}_{1}\right)$ into the crisp linear programming problem $\left(\mathrm{P}_{2}\right)$, to find the fuzzy optimal solution.

$\operatorname{Max}\left(\right.$ or Min) $\quad \mathfrak{R}\left(\tilde{C}^{T} \otimes \tilde{X}\right)$

Subject to

$$
\begin{gathered}
\mathfrak{R}(\tilde{A} \otimes \tilde{X}) \leq \text { or }=\text { or } \geq \mathfrak{R}(\tilde{b}) \\
\beta_{j 1}-\beta_{j 2} \geq 0, \beta_{j 2} \geq 0, \beta_{j 3} \geq 0, \beta_{j 4}-\beta_{j 3} \geq 0 ; \\
j=1,2, \ldots, n
\end{gathered}
$$

or

Max (or Min)

$$
\left(\frac{1}{16} \sum_{j=1}^{n}\left[\begin{array}{l}
\left(16 c_{j}+\delta_{j 3}-\delta_{j 2}+(4-3 \lambda)\left(\delta_{j 4}\right.\right. \\
\left.\left.-\delta_{j 1}\right)\right) m_{j}+\beta_{j 1}(4-3 \lambda)\left(c_{j}+\delta_{j 1}\right. \\
) \beta_{j 2}\left(\delta_{j 2}-c_{j}\right)+\beta_{j 3}\left(c_{j}+\delta_{j 3}\right)+ \\
\beta_{j 4}(4-3 \lambda)\left(c_{j}+\delta_{j 4}\right)
\end{array}\right]\right)
$$

Subject to

$$
\begin{aligned}
& \sum_{j=1}^{n}\left[\begin{array}{l}
\left(16 a_{k j}+\delta_{k j 3}-\delta_{k j 2}+(4-3 \lambda)\left(\delta_{k j 4}-\delta_{k j 1}\right)\right) \\
m_{j}+\beta_{j 1}(4-3 \lambda)\left(a_{k j}+\delta_{j k 1}\right) \beta_{j 2}\left(\delta_{k j 2}-a_{k j}\right) \\
+\beta_{j 3}\left(a_{k j}+\delta_{k j 3}\right)+\beta_{j 4}(4-3 \lambda)\left(a_{k j}+\delta_{k j 4}\right)
\end{array}\right] \\
& \leq \text { or }=\text { or } \geq\left[16 b_{k}+\alpha_{k 3}-\alpha_{k 2}+(4-3 \lambda)\left(\alpha_{k 4}-\right.\right. \\
& \left.\left.\alpha_{k 1}\right)\right], k=1,2, \ldots, m \\
& \beta_{j 1}-\beta_{j 2} \geq 0, \beta_{j 2} \geq 0, \beta_{j 3} \geq 0, \beta_{j 4}-\beta_{j 3} \geq 0 \\
& j=1,2, \ldots, n
\end{aligned}
$$

Step 2 Solve the crisp linear programming problem $\left(\mathrm{P}_{2}\right)$ to find the optimal solution

$$
\left\{m_{j}, \beta_{j 1}, \beta_{j 2}, \beta_{j 3}, \beta_{j 4} ; j=1,2, \ldots, n\right\} .
$$

Step 3 Find the fuzzy optimal value of the chosen interval-valued fully fuzzy linear programming problem $\left(\mathrm{P}_{1}\right)$ by substituting the values of fuzzy optimal solutions

$$
\begin{gathered}
\tilde{X}=\left[\tilde{x}_{j}=\left[\left(m_{j}-\beta_{j 2}, m_{j}, m_{j}+\beta_{j 3} ; \lambda\right),\left(m_{j}-\beta_{j 1}\right.\right.\right. \\
\left.\left.\left., m_{j}, m_{j}+\beta_{j 4} ; 1\right)\right]\right]_{n \times 1}, \text { in } \tilde{C}^{T} \otimes \tilde{X} .
\end{gathered}
$$

Step 4 If any change is made in the chosen interval-valued fully fuzzy linear programming problem $\left(\mathrm{P}_{1}\right)$ then the optimal solution of the resulting interval-valued fully fuzzy linear programming problem can be obtained as follows:

Case 1: A change in the fuzzy cost vector

If the cost vector $\tilde{C}^{T}$ changes to $\tilde{C}^{{ }^{T}}$ in the original interval-valued fully fuzzy linear programming problem $\left(\mathrm{P}_{1}\right)$ then replace $\mathfrak{R}\left(\tilde{C}^{T} \otimes \tilde{X}\right)$ by $\mathfrak{R}\left(\tilde{C}^{{ }^{T}} \otimes \tilde{X}\right)$ in crisp linear programming $\left(\mathrm{P}_{2}\right)$ to obtain $\left(\mathrm{P}_{3}\right)$

$\operatorname{Max}\left(\right.$ or Min) $\quad \mathfrak{R}\left(\tilde{C}^{T T} \otimes \tilde{X}\right) \quad\left(\mathrm{P}_{3}\right)$

Subject to

$$
\mathfrak{R}(\tilde{A} \otimes \tilde{X}) \leq \text { or }=\text { or } \geq \mathfrak{R}(\tilde{b})
$$

$$
\begin{aligned}
& \beta_{j 1}-\beta_{j 2} \geq 0, \beta_{j 2} \geq 0, \beta_{j 3} \geq 0, \beta_{j 4}-\beta_{j 3} \geq 0 \\
& j=1,2, \ldots, n
\end{aligned}
$$

\section{Case 2: Change in fuzzy requirement vector $\tilde{b}$}

If the change in fuzzy requirement vector is made i.e., $\tilde{b}$ is changed to $\tilde{b}^{\prime}$ in the original intervalvalued fully fuzzy linear programming problem $\left(\mathrm{P}_{1}\right)$ then, replace $\mathfrak{R}(\tilde{b})$ by $\mathfrak{R}\left(\tilde{b}^{\prime}\right)$ in the crisp linear programming problem $\left(\mathrm{P}_{2}\right)$ to obtain $\left(\mathrm{P}_{4}\right)$ :

\section{$\operatorname{Max}\left(\right.$ or Min) $\quad \mathfrak{R}\left(\tilde{C}^{T} \otimes \tilde{X}\right)$}

Subject to

$$
\begin{gathered}
\mathfrak{R}(\tilde{A} \otimes \tilde{X}) \leq \text { or }=\text { or } \geq \mathfrak{R}\left(\tilde{b}^{\prime}\right) \\
\beta_{j 1}-\beta_{j 2} \geq 0, \beta_{j 2} \geq 0, \beta_{j 3} \geq 0, \beta_{j 4}-\beta_{j 3} \geq 0 \\
j=1,2, \ldots, n
\end{gathered}
$$




\section{Case 3: Addition of a new fuzzy variable}

Let us suppose that a new non-negative fuzzy variable, say $\tilde{x}_{n+1}$, is added in original intervalvalued fully fuzzy linear programming problem $\left(\mathrm{P}_{1}\right)$ Assume that if $\tilde{c}_{n+1}$ is the cost and $\tilde{A}_{n+1}$ is the column associated with $\tilde{X}_{n+1}$, then replace $\mathfrak{R}(\tilde{A} \otimes \tilde{X}) \quad$ by $\quad \mathfrak{R}\left(\tilde{A} \otimes \tilde{X} \oplus \tilde{A}_{n+1} \otimes \tilde{X}_{n+1}\right) \quad$ and $\mathfrak{R}\left(\tilde{C}^{T} \otimes \tilde{X}\right)$ by $\mathfrak{R}\left(\tilde{C}^{T} \otimes \tilde{X} \oplus \tilde{C}_{n+1} \otimes \tilde{X}_{n+1}\right)$ in $\left(\mathrm{P}_{2}\right)$ to obtain the crisp linear programming problem $\left(\mathrm{P}_{5}\right)$ :

$\operatorname{Max}\left(\right.$ or Min) $\quad \mathfrak{R}\left(\tilde{C}^{T} \otimes \tilde{X} \oplus \tilde{C}_{n+1} \otimes \tilde{X}_{n+1}\right)$

Subject to

$$
\begin{gathered}
\Re\left(\tilde{A} \otimes \tilde{X} \oplus \tilde{A}_{n+1} \otimes \tilde{x}_{n+1}\right) \leq \text { or }=\text { or } \geq \Re(\tilde{b}) \\
\beta_{j 1}-\beta_{j 2} \geq 0, \beta_{j 2} \geq 0, \beta_{j 3} \geq 0, \beta_{j 4}-\beta_{j 3} \geq 0 ; \\
j=1,2, \ldots, n
\end{gathered}
$$

\section{Case 4: Change in fuzzy constraint matrix}

Let us suppose that the column of the constraint matrix, corresponding to the fuzzy variable $\tilde{x}_{j}$, is changed from $\tilde{A}_{j}$ to $\tilde{A}_{j}^{\prime}$ in the original intervalvalued fully fuzzy linear programming problem then, replace $\quad \mathfrak{R}(\tilde{A} \otimes \tilde{X}) \leq$ or $=$ or $\geq \mathfrak{R}(\tilde{b}) \quad$ by $\mathfrak{R}\left(\tilde{A}^{\prime} \otimes \tilde{X}\right) \leq$ or $=$ or $\geq \mathfrak{R}(\tilde{b})$ in $\left(\mathrm{P}_{2}\right)$ to obtain a new crisp linear programming problem $\left(\mathrm{P}_{6}\right)$ :

$\operatorname{Max}\left(\right.$ or Min) $\quad \Re\left(\tilde{C}^{T} \otimes \tilde{X}\right)$

Subject to

$$
\begin{gathered}
\mathfrak{R}\left(\tilde{A}^{\prime} \otimes \tilde{X}\right) \leq \text { or }=\text { or } \geq \mathfrak{R}(\tilde{b}) \\
\beta_{j 1}-\beta_{j 2} \geq 0, \beta_{j 2} \geq 0, \beta_{j 3} \geq 0, \beta_{j 4}-\beta_{j 3} \geq 0 ; \\
j=1,2, \ldots, n
\end{gathered}
$$

\section{Case 5: Addition of a new fuzzy constraint}

Let us suppose that a new fuzzy constraint is added in the original interval-valued fully fuzzy linear programming problem $\left(\mathrm{P}_{1}\right)$ then, replace $\mathfrak{R}(\tilde{A} \otimes \tilde{X}) \leq$ or $=$ or $\geq \mathfrak{R}(\tilde{b}) \quad$ by $\quad \mathfrak{R}\left(\tilde{A}^{\prime} \otimes \tilde{X}\right) \leq$ or $=$ or $\geq \mathfrak{R}\left(\tilde{b}^{\prime}\right)$ in $\left(\mathrm{P}_{2}\right)$ to obtain a crisp linear programming problem $\left(\mathrm{P}_{7}\right)$

$\operatorname{Max}\left(\right.$ or Min) $\quad \mathfrak{R}\left(\tilde{C}^{T} \otimes \tilde{X}\right)$

Subject to

$$
\begin{gathered}
\Re\left(\tilde{A}^{\prime} \otimes \tilde{X}\right) \leq \text { or }=\text { or } \geq \mathfrak{R}\left(\tilde{b}^{\prime}\right) \\
\beta_{j 1}-\beta_{j 2} \geq 0, \beta_{j 2} \geq 0, \beta_{j 3} \geq 0, \beta_{j 4}-\beta_{j 3} \geq 0 ; \\
j=1,2, \ldots, n
\end{gathered}
$$

Step 4 Now, apply the existing sensitivity analysis technique to find the fuzzy optimal solutions of $\left(\mathrm{P}_{3}\right),\left(\mathrm{P}_{4}\right),\left(\mathrm{P}_{5}\right),\left(\mathrm{P}_{6}\right)$ and $\left(\mathrm{P}_{7}\right)$ with the help of an optimal solution of $\left(\mathrm{P}_{2}\right)$ and use the step 3 of the proposed method to find the fuzzy optimal solution and the fuzzy optimal value of the resulting interval-valued fully fuzzy linear programming problem.

Remark 3.1 The other cases i.e., deletion of fuzzy variables, deletion of fuzzy constraints, simultaneous change in coefficients of the fuzzy decision variables in objective function and fuzzy requirement vectors etc. can also be solved by using the proposed method.

\section{Numerical example}

In this section, the proposed method is illustrated with the help of a numerical example:

\section{Example 4.1}

A factory produces automobiles and trucks, each one requires three processes. The production condition is shown in Table 1.

\begin{tabular}{|l|l|l|l|l|}
\hline Type & $\begin{array}{l}\text { Process } \\
1(\mathrm{hr} .)\end{array}$ & $\begin{array}{l}\text { Process } \\
2(\mathrm{hr} .)\end{array}$ & $\begin{array}{l}\text { Process } \\
3(\mathrm{hr} .)\end{array}$ & $\begin{array}{l}\text { Profit } \\
(100 \\
\$)\end{array}$ \\
\hline Automob. & 15 & 24 & 21 & 25 \\
\hline Truck & 30 & 6 & 14 & 48 \\
\hline $\begin{array}{l}\text { Total } \\
\text { hour }\end{array}$ & 450 & 240 & 280 & \\
\hline
\end{tabular}

Table 1. Production condition 
Assuming the quantity of automobiles and truck produced as $x_{1}$ and $x_{2}$. Then the chosen problem can be formulated into the following crisp linear programming problem:

Maximize $\quad\left[25 x_{1}+48 x_{2}\right]$

Subject to

$$
\begin{gathered}
15 x_{1}+30 x_{2} \leq 450 \\
24 x_{1}+6 x_{2} \leq 240 \\
21 x_{1}+14 x_{2} \leq 280 \\
x_{1} \geq 0, x_{2} \geq 0
\end{gathered}
$$

Assuming

$\delta_{11}=7, \delta_{12}=6, \delta_{13}=8, \delta_{14}=9, \delta_{21}=5, \delta_{22}=4, \delta_{23}=6$,

$\delta_{24}=8, \delta_{111}=5, \delta_{112}=1, \delta_{113}=2, \delta_{114}=3, \delta_{121}=7, \delta_{122}$

$=5, \delta_{123}=4, \delta_{124}=8, \delta_{211}=4, \delta_{212}=3, \delta_{213}=3, \delta_{214}=$

9, $\delta_{221}=4, \delta_{222}=2, \delta_{223}=2, \delta_{224}=5, \delta_{311}=5, \delta_{312}=4$,

$\delta_{313}=1, \delta_{314}=5, \delta_{321}=6, \delta_{322}=2, \delta_{323}=5, \delta_{324}=8, \alpha_{11}$

$=30, \alpha_{12}=20, \alpha_{13}=30, \alpha_{14}=70, \alpha_{21}=60, \alpha_{22}=20$,

$\alpha_{23}=50, \alpha_{24}=60, \alpha_{31}=50, \alpha_{32}=10, \alpha_{33}=30, \alpha_{34}=$

$40, \delta_{411}=4, \delta_{412}=3, \delta_{413}=2, \delta_{414}=9, \delta_{421}=4, \delta_{422}=$

$2, \delta_{423}=2, \delta_{424}=5, \alpha_{41}=60, \alpha_{42}=20, \alpha_{43}=50, \alpha_{44}$

$=60, \lambda=.9, \tilde{x}_{j}=\left[\left(m_{j}-\beta_{j 2}, m_{j}, m_{j}+\beta_{j 3} ; .9\right),\left(m_{j}\right.\right.$

$\left.\left.-\beta_{j 1}, m_{j}, m_{j}+\beta_{j 4} ; 1\right)\right] j=1,2,3$

the crisp linear programming problem $\left(\mathrm{P}_{8}\right)$ is converted into interval-valued fuzzy linear programming problem $\left(\mathrm{P}_{9}\right)$

$\operatorname{Max}$

$$
\begin{aligned}
& {\left[[(19,25,33 ; .9),(18,25,34 ; 1)] \otimes \tilde{x}_{1} \oplus[(44,\right.} \\
& \left.\quad 48,54 ; .9),(43,48,56 ; 1)] \otimes \tilde{x}_{2}\right]
\end{aligned}
$$

Subject to

$[(14,15,17 ; .9),(10,15,18 ; 1)] \otimes \tilde{x}_{1} \oplus[(25,30,34 ; .9),(23$, $30,38 ; 1)] \otimes \tilde{x}_{2} \leq[(430,450,480 ; .9),(420,450,520 ; 1)]$
$[(21,24,26 ; .9),(20,24,33 ; 1)] \otimes \tilde{x}_{1} \oplus[(4,6,8 ; .9),(2,6$, $11 ; 1)] \otimes \tilde{x}_{2} \leq[(220,240,290 ; .9),(180,240,300 ; 1)]$

$[(17,21,22 ; .9),(16,21,26 ; 1)] \otimes \tilde{x}_{1} \oplus[(12,14,19 ; .9),(8$, $14,22 ; 1)] \otimes \tilde{x}_{2} \leq[(270,280,310 ; .9),(230,280,320 ; 1)]$

$\tilde{x}_{1}, \tilde{x}_{2}$ are non negative interval-valued fuzzy numbers.

a) Discuss the effect of changing the cost coefficients $[(19,25,33 ; .9),(18,25,34 ; 1)] \quad$ and $[(44,48,54 ; .9),(43,48,56 ; 1)]$ of the fuzzy decision variables $\tilde{x}_{1}$ and $\tilde{x}_{2}$ to $[(22,28,36 ; .9),(21,28,37 ; 1)]$ and $[(46,50,56$;

$.9),(45,50,58 ; 1)]$ respectively on the fuzzy optimal solution and fuzzy optimal value of resulting interval-valued fully fuzzy linear programming problem.

b) Discuss the effect of changing the fuzzy requirement vector from [(430, 450, 480;.9), (420, 450, 520;1)],[(220, 240,

$290 ; .9),(180,240,300 ; 1)] \quad$ and $\quad[(270,280$

,310;.9), $(230,280,320 ; 1)] \quad$ to $\quad[(480,500$,

530;.9), $(470,500,570 ; 1)], \quad[(210,230,280 ; .9$

)$,(170,230,290 ; 1)] \quad$ and $\quad[(290,300,330 ; .9)$,

$(250,300,340 ; 1)]$ on the fuzzy optimal solution and fuzzy optimal value of the resulting interval-valued fully fuzzy linear programming problem.

c) Find the effect of adding a new non-negative fuzzy variable $\tilde{x}_{3}$ with cost $[(46,50,56 ; .9),(45,50,58 ; 1)] \quad$ and $\quad[[(14,15$, 17;.9),(10,15,18;1)], [(21, 24,26;.9),(20,24,33;1)],

$[(14,15,17 ; .9),(10,15,18 ; 1)]]^{T}$ as column vectors on the fuzzy optimal solution and fuzzy optimal value of resulting interval-valued fully fuzzy linear programming problem.

d) Discuss the effect of changing the column of the constraint matrix corresponding to the fuzzy variable $\tilde{x}_{2}$ to [[(30,35,39;.9), $(28,35,43 ; 1)],[(3,5,7 ; .9)$, 
$(1,5,10 ; 1)],[(13,15,20 ; .9),(9,15,23 ; 1)]]^{T}$ on the fuzzy optimal solution and fuzzy optimal value of resulting interval-valued fully fuzzy linear programming problem.

e) Find the effect of adding a new fuzzy constraint $[(18,21,23 ; .9),(17,21,30 ; 1)] \otimes \tilde{x}_{1}$

$\oplus[(18,20,22 ; .9),(16,20,25 ; 1)] \otimes \tilde{x}_{2} \leq[(480,500$

,550;.9),(400,500500,560;1)] on the fuzzy optimal solution and fuzzy optimal value of resulting interval-valued fully fuzzy linear programming problem.

Solution: The solution of the interval-valued fully fuzzy sensitivity analysis problem, chosen in example 4.1, can be obtained by using the following steps of the proposed method:

Step 1 As discussed in step 1 of the proposed method, to find the fuzzy optimal solution of the chosen interval-valued fully fuzzy linear programming problem $\left(\mathrm{P}_{9}\right)$ first convert $\left(\mathrm{P}_{9}\right)$ into crisp linear programming problem $\left(\mathrm{P}_{10}\right)$

$\operatorname{Max} \frac{1}{16}\left[\begin{array}{l}404.6 m_{1}-23 \beta_{11}-19 \beta_{12}+33 \beta_{13}+44.2 \beta_{14}+ \\ 773.9 m_{2}-55.9 \beta_{21}-44 \beta_{22}+52 \beta_{23}+72.8 \beta_{24}\end{array}\right]$

Subject to $\quad\left(\mathrm{P}_{10}\right)$

$$
\begin{aligned}
& 238.4 m_{1}+26 \beta_{11}-14 \beta_{12}+17 \beta_{13}+23.4 \beta_{14}+480.3 m_{2} \\
& +48.1 \beta_{21}-25 \beta_{22}+34 \beta_{23}+49.4 \beta_{24} \leq 453.875 \\
& 389.5 m_{1}+36.4 \beta_{11}-21 \beta_{12}+26 \beta_{13}+42.9 \beta_{14}+97.3 \\
& m_{2}+13 \beta_{21}-4 \beta_{22}+8 \beta_{23}+14.3 \beta_{24} \leq 241.875 \\
& 333 m_{1}+33.8 \beta_{11}-17 \beta_{12}+22 \beta_{13}+33.8 \beta_{14}+229.6 \\
& m_{2}+26 \beta_{21}-12 \beta_{22}+19 \beta_{23}+28.6 \beta_{24} \leq 280.4375 \\
& \beta_{j 1}-\beta_{j 2} \geq 0, \beta_{j 2} \geq 0, \beta_{j 3} \geq 0, \beta_{j 4}-\beta_{j 3} \geq 0 ; j=1,2 .
\end{aligned}
$$

Step 2 The optimal solution of the crisp linear programming problem $\left(\mathrm{P}_{10}\right)$ is

$$
\begin{aligned}
& m_{1}=0, m_{2}=0.8581, \beta_{11}=\beta_{12}=\beta_{13}=\beta_{14}=\beta_{21}= \\
& \beta_{22}=\beta_{23}=0, \beta_{24}=2.916 .
\end{aligned}
$$

Step 3 Substituting these values of $\left\{m_{1}, m_{2}, \beta_{11}, \beta_{12}, \beta_{13}, \beta_{14}, \beta_{21}, \beta_{22}, \beta_{23}, \beta_{24}\right\} \quad$ in $\tilde{x}_{j}=\left[\left(m_{j}-\beta_{j 2}, m_{j}, m_{j}+\beta_{j 3} ; .9\right),\left(m_{j}-\beta_{j 1}, m_{j}, m_{j}\right.\right.$ $\left.\left.+\beta_{j 4} ; 1\right)\right] ; j=1,2$ the fuzzy optimal solution of chosen the interval valued fully fuzzy linear programming problem is

$\tilde{x}_{1}=[(0,0,0 ; .9),(0,0,0 ; 1)], \tilde{x}_{2}=[(.8581, .8581, .8581 ;$

$.9),(.8581, .8581,3.7741 ; 1)]$ and putting $\tilde{x}_{1}, \tilde{x}_{2}$ in $\left[[(19,25,33 ; .9),(18,25,34 ; 1)] \otimes \tilde{x}_{1} \oplus[(44,48,54 ; .9)\right.$,

$\left.(43,48,56 ; 1)] \otimes \tilde{x}_{2}\right]$ the fuzzy optimal value is

\section{[(3.7756, 4.2518, 4.7833;.9), (3.8089, 4.2518, 4.9605;1)].}

a) Since the cost coefficients corresponding to the fuzzy variables $\tilde{x}_{1}$ and $\tilde{x}_{2}$ change to [(22, 28,36;.9), $(21,28,37 ; 1)] \quad$ and $[(46,50,56 ; .9),(45,50, \quad 58 ; 1)]$ respectively in the original interval-valued fully fuzzy linear programming problem $\left(\mathrm{P}_{9}\right)$ so replacing crisp linear programming problem $\left(\mathrm{P}_{10}\right)$ by $\left(\mathrm{P}_{11}\right)$

Max

$$
\frac{1}{16}\left[\begin{array}{l}
452.6 m_{1}+27.3 \beta_{11}-22 \beta_{12}+36 \beta_{13}+39 \beta_{14}+ \\
805.9 m_{2}+58.5 \beta_{21}-46 \beta_{22}+56 \beta_{23}+75.4 \beta_{24}
\end{array}\right]
$$

Subject to $\quad\left(\mathrm{P}_{11}\right)$

$$
\begin{gathered}
238.4 m_{1}+26 \beta_{11}-14 \beta_{12}+17 \beta_{13}+23.4 \beta_{14}+480.3 \\
m_{2}+48.1 \beta_{21}-25 \beta_{22}+34 \beta_{23}+49.4 \beta_{24} \leq 453.875 \\
389.5 m_{1}+36.4 \beta_{11}-21 \beta_{12}+26 \beta_{13}+42.9 \beta_{14}+97.3 \\
m_{2}+13 \beta_{21}-4 \beta_{22}+8 \beta_{23}+14.3 \beta_{24} \leq 241.875 \\
333 m_{1}+33.8 \beta_{11}-17 \beta_{12}+22 \beta_{13}+33.8 \beta_{14}+229.6 \\
m_{2}+26 \beta_{21}-12 \beta_{22}+19 \beta_{23}+28.6 \beta_{24} \leq 280.4375 \\
\beta_{j 1}-\beta_{j 2} \geq 0, \beta_{j 2} \geq 0, \beta_{j 3} \geq 0, \beta_{j 4}-\beta_{j 3} \geq 0 ; j=1,2 .
\end{gathered}
$$

Now, applying the existing sensitivity analysis technique the optimal solution of crisp linear programming problem $\left(\mathrm{P}_{11}\right)$ is: 


$$
\begin{aligned}
& m_{1}=0, m_{2}=0.8581, \beta_{11}=\beta_{12}=\beta_{13}=\beta_{14}=\beta_{21}= \\
& \beta_{22}=\beta_{23}=0, \beta_{24}=2.916 .
\end{aligned}
$$

Using Step 3 of the proposed method the fuzzy optimal value of resulting interval-valued fully fuzzy linear programming problem is

\section{[(3.947, 4.2905, 4.8053;.9), (3.8614, 4.2905,} 4.9769;1)].

b) Since the fuzzy requirement vector $\tilde{b}$ has changed from [(430,450,480;.9),(420,450, 520;1)], and $\quad[(270,280,310 ; .9),(230,280,320 ; 1)]$ to [(480,500, 530;.9), (470,500,570;1)],[ $(210,230,280 ; .9),(170,230,290 ; 1)] \quad$ and $\quad[(290$, $300,330 ; .9),(250,300,340 ; 1)]$ respectively in the original interval-valued fully fuzzy linear programming problem $\left(\mathrm{P}_{9}\right)$ so replacing the crisp linear programming problem $\left(\mathrm{P}_{10}\right)$ by $\left(\mathrm{P}_{12}\right)$

$\operatorname{Max}$

$$
\frac{1}{16}\left[\begin{array}{l}
404.6 m_{1}-23 \beta_{11}-19 \beta_{12}+33 \beta_{13}+44.2 \beta_{14}+ \\
773.9 m_{2}-55.9 \beta_{21}-44 \beta_{22}+52 \beta_{23}+72.8 \beta_{24}
\end{array}\right]
$$

Subject to

$\left(\mathrm{P}_{12}\right)$

$$
\begin{aligned}
& 238.4 m_{1}+26 \beta_{11}-14 \beta_{12}+17 \beta_{13}+23.4 \beta_{14}+480.3 \\
& m_{2}+48.1 \beta_{21}-25 \beta_{22}+34 \beta_{23}+49.4 \beta_{24} \leq 503.875 \\
& 389.5 m_{1}+36.4 \beta_{11}-21 \beta_{12}+26 \beta_{13}+42.9 \beta_{14}+97.3 \\
& m_{2}+13 \beta_{21}-4 \beta_{22}+8 \beta_{23}+14.3 \beta_{24} \leq 231.875 \\
& 333 m_{1}+33.8 \beta_{11}-17 \beta_{12}+22 \beta_{13}+33.8 \beta_{14}+229.6 \\
& m_{2}+26 \beta_{21}-12 \beta_{22}+19 \beta_{23}+28.6 \beta_{24} \leq 300.4375 \\
& \beta_{j 1}-\beta_{j 2} \geq 0, \beta_{j 2} \geq 0, \beta_{j 3} \geq 0, \beta_{j 4}-\beta_{j 3} \geq 0 ; \\
& j=1,2 .
\end{aligned}
$$

Now, applying the existing sensitivity analysis technique, the optimal solution of crisp linear programming problem $\left(\mathrm{P}_{12}\right)$ is

$$
\begin{aligned}
& m_{1}=0, m_{2}=0.9675, \beta_{11}=\beta_{12}=\beta_{13}=\beta_{14}=\beta_{21}= \\
& \beta_{22}=\beta_{23}=0, \beta_{24}=2.7369
\end{aligned}
$$

Using Step 3 of the proposed method the fuzzy optimal value of resulting interval-valued fully fuzzy linear programming problem is:

[(4.2570, 4.6440, 5.2245;.9), (4.1602, 4.6440, $5.4180 ; 1)]$

c) Since a new non-negative interval-valued fuzzy variable $\quad \tilde{x}_{3} \quad$ with fuzzy cost $[(46,50,56 ; .9),(45,50,58 ; 1)]$ and column vectors [[(14,15,17;.9), (10,15,18;1)],[(21,

24, 26;.9),(20,24,33;1)],[(14,15,17;.9),(10,15, ,18;1)] $]^{T}$ is added to the original interval- valued fully fuzzy linear programming problem $\left(\mathrm{P}_{9}\right)$ so replace the crisp linear programming problem $\left(\mathrm{P}_{10}\right)$ into the crisp linear programming problem $\left(\mathrm{P}_{13}\right)$

$\operatorname{Max}$

$\frac{1}{16}\left[\begin{array}{l}404.6 m_{1}-23 \beta_{11}-19 \beta_{12}+33 \beta_{13}+44.2 \beta_{14}+ \\ 773.9 m_{2}-55.9 \beta_{21}-44 \beta_{22}+52 \beta_{23}+72.8 \beta_{24} \\ +805.9 m_{3}+58.5 \beta_{31}-46 \beta_{32}+56 \beta_{33}+75.4 \beta_{34}\end{array}\right]$

Subject to $\left(\mathrm{P}_{13}\right)$

$$
\begin{aligned}
& 238.4 m_{1}+26 \beta_{11}-14 \beta_{12}+17 \beta_{13}+23.4 \beta_{14}+480.3 \\
& m_{2}+48.1 \beta_{21}-25 \beta_{22}+34 \beta_{23}+49.4 \beta_{24}+389.5 m_{3} \\
& +36.4 \beta_{31}-21 \beta_{32}+26 \beta_{33}+42.9 \beta_{34} \leq 453.875
\end{aligned}
$$$$
389.5 m_{1}+36.4 \beta_{11}-21 \beta_{12}+26 \beta_{13}+42.9 \beta_{14}+97.3
$$$$
m_{2}+13 \beta_{21}-4 \beta_{22}+8 \beta_{23}+14.3 \beta_{24}+238.4 m_{3}+26 \beta_{31}
$$$$
-14 \beta_{32}+17 \beta_{33}+23.4 \beta_{34} \leq 241.875
$$$$
333 m_{1}+33.8 \beta_{11}-17 \beta_{12}+22 \beta_{13}+33.8 \beta_{14}+229.6
$$$$
m_{2}+26 \beta_{21}-12 \beta_{22}+19 \beta_{23}+28.6 \beta_{24}+389.5 m_{3}+
$$$$
36.4 \beta_{31}-21 \beta_{32}+26 \beta_{33}+42.9 \beta_{34} \leq 280.4375
$$

$$
\begin{aligned}
& \beta_{j 1}-\beta_{j 2} \geq 0, \beta_{j 2} \geq 0, \beta_{j 3} \geq 0, \beta_{j 4}-\beta_{j 3} \geq 0 \\
& j=1,2,3 .
\end{aligned}
$$


Now, applying the existing sensitivity analysis technique, the optimal solution of crisp linear programming problem $\left(\mathrm{P}_{13}\right)$ is

$$
\begin{aligned}
& m_{1}=m_{3}=0, m_{2}=0.8581, \beta_{11}=\beta_{12}=\beta_{13}=\beta_{14}= \\
& \beta_{21}=\beta_{22}=\beta_{23}=\beta_{31}=\beta_{32}=\beta_{33}=\beta_{34}=0, \beta_{24} \\
& =2.916
\end{aligned}
$$

Using Step 3 of the proposed method the fuzzy optimal value of resulting interval-valued fully fuzzy linear programming problem is:

[(3.7756, 4.2518, 4.7833;.9), (3.8089, 4.2518, 4.9605;1)]

d) Since the column of the constraint matrix corresponding to the fuzzy decision variable $\tilde{x}_{2}$ has changed to [[(30,35,39;.9), (28,35,43;1)] ,[(3,5, 7;.9), (1, 5,10;1)],[(13,15, 20;.9), $(9,15,23 ; 1)]]^{T}$ in the original interval-valued fully fuzzy linear programming problem $\left(\mathrm{P}_{9}\right)$ so replacing the crisp linear programming problem $\left(\mathrm{P}_{10}\right)$ by crisp linear programming problem $\left(\mathrm{P}_{14}\right)$

Max

$$
\frac{1}{16}\left[\begin{array}{l}
404.6 m_{1}-23 \beta_{11}-19 \beta_{12}+33 \beta_{13}+44.2 \beta_{14}+ \\
773.9 m_{2}-55.9 \beta_{21}-44 \beta_{22}+52 \beta_{23}+72.8 \beta_{24}
\end{array}\right]
$$

Subject to

$$
\left(\mathrm{P}_{14}\right)
$$

$$
\begin{aligned}
& 238.4 m_{1}+26 \beta_{11}-14 \beta_{12}+17 \beta_{13}+23.4 \beta_{14}+560.3 \\
& m_{2}+54.6 \beta_{21}+30 \beta_{22}+39 \beta_{23}+55.9 \beta_{24} \leq 453.875 \\
& 389.5 m_{1}+36.4 \beta_{11}-21 \beta_{12}+26 \beta_{13}+42.9 \beta_{14}+81.3 \\
& m_{2}+11.7 \beta_{21}-3 \beta_{22}-3 \beta_{23}+13 \beta_{24} \leq 241.875 \\
& 333 m_{1}+33.8 \beta_{11}-17 \beta_{12}+22 \beta_{13}+33.8 \beta_{14}+245.6 \\
& m_{2}+25.5 \beta_{21}-13 \beta_{22}+20 \beta_{23}+29.9 \beta_{24} \leq 280.4375 \\
& \quad \beta_{j 1}-\beta_{j 2} \geq 0, \beta_{j 2} \geq 0, \beta_{j 3} \geq 0, \beta_{j 4}-\beta_{j 3} \geq 0 \\
& \quad ; j=1,2 .
\end{aligned}
$$

Now, apply the existing sensitivity analysis technique, and the optimal solution of crisp linear programming problem $\left(\mathrm{P}_{14}\right)$ is

$$
m_{1}=0, m_{2}=0.7836, \beta_{11}=\beta_{12}=\beta_{21}=\beta_{22}=\beta_{23}=
$$$$
\beta_{24}=0, \beta_{13}=\beta_{14}=1.5767
$$

step 3 of the proposed method the fuzzy optimal value of resulting interval-valued fully fuzzy linear programming problem is:

[(3.4478, 3.7612, 4.2314;.9), (3.3694, 3.7612, $4.3881 ; 1)]$.

e) Since a new fuzzy constraint $[(18,21,23 ; .9),(17,21,30 ; 1)] \otimes \tilde{x}_{1} \oplus[(18,20,22 ; .9),(16$ $, 20,25 ; 1)] \otimes \tilde{x}_{2} \leq[(480,500,550 ; .9),(440,500,560 ; 1)] \quad$ is added to the original interval-valued fully fuzzy linear programming problem $\left(\mathrm{P}_{9}\right)$ so replacing the crisp linear programming problem $\left(\mathrm{P}_{10}\right)$ by crisp linear programming problem $\left(\mathrm{P}_{15}\right)$

Max

$\frac{1}{16}\left[\begin{array}{l}404.6 m_{1}-23 \beta_{11}-19 \beta_{12}+33 \beta_{13}+44.2 \beta_{14}+ \\ 773.9 m_{2}-55.9 \beta_{21}-44 \beta_{22}+52 \beta_{23}+72.8 \beta_{24}\end{array}\right]$

Subject to

$\left(\mathrm{P}_{15}\right)$

$238.4 m_{1}+26 \beta_{11}-14 \beta_{12}+17 \beta_{13}+23.4 \beta_{14}+480.3$

$m_{2}+48.1 \beta_{21}-25 \beta_{22}+34 \beta_{23}+49.4 \beta_{24} \leq 453.875$

$389.5 m_{1}+36.4 \beta_{11}-21 \beta_{12}+26 \beta_{13}+42.9 \beta_{14}+97.3$

$m_{2}+13 \beta_{21}-4 \beta_{22}+8 \beta_{23}+14.3 \beta_{24} \leq 241.875$

$333 m_{1}+33.8 \beta_{11}-17 \beta_{12}+22 \beta_{13}+33.8 \beta_{14}+229.6$

$m_{2}+26 \beta_{21}-12 \beta_{22}+19 \beta_{23}+28.6 \beta_{24} \leq 280.4375$

$340.2 m_{1}+32.5 \beta_{11}-18 \beta_{12}+23 \beta_{13}+39 \beta_{14}+321.3$

$m_{2}+31.2 \beta_{21}-18 \beta_{22}+22 \beta_{23}+32.5 \beta_{24} \leq 501.875$

$\beta_{j 1}-\beta_{j 2} \geq 0, \beta_{j 2} \geq 0, \beta_{j 3} \geq 0, \beta_{j 4}-\beta_{j 3} \geq 0 ; j=1,2$ 
Now, applying the existing sensitivity analysis technique, the optimal solution of crisp linear programming problem $\left(\mathrm{P}_{15}\right)$ is:

$$
\begin{aligned}
& m_{1}=m_{3}=0, m_{2}=0.8581, \beta_{11}=\beta_{12}=\beta_{13}=\beta_{14}=\beta_{21}= \\
& \beta_{22}=\beta_{23}=\beta_{31}=\beta_{32}=\beta_{33}=\beta_{34}=0, \beta_{24}=2.916
\end{aligned}
$$

Using step 3 of the proposed method, the fuzzy optimal value of the resulting interval-valued fully fuzzy linear programming problem is,

\section{[(3.7756, 4.2518, 4.7833;.9), (3.8089, 4.2518,}

$4.9605 ; 1)]$

\subsection{Physical interpretation of the results}

In this section, the effect of changing the cost coefficients on the results is physically interpreted. Similarly, the results of other cases, presented in table 2 , can also be physically interpreted.
From the results, obtained by using original interval-valued fully fuzzy linear programming problem and resulting interval-valued fully fuzzy linear programming problem, it can be easily seen that in Case 1:

a) For the original interval-valued fully fuzzy linear programming problem the optimal value will be greater than and lesser than units while for the interval-valued fully fuzzy linear programming problem obtained by changing the cost coefficients corresponding to the fuzzy decision variables and the optimal value will be greater than and lesser than units.

b) For the original interval-valued fully fuzzy linear programming problem, the overall level of satisfaction of the decision maker about the statement that the optimal value will be units lies in and for the interval-valued fully fuzzy linear programming problem, obtained by changing the

\begin{tabular}{|l|l|l|}
\hline & $\begin{array}{l}\text { Fuzzy optimal value obtained } \\
\text { from original interval-valued fully } \\
\text { fuzzy linear programming } \\
\text { problem }\end{array}$ & $\begin{array}{l}\text { Fuzzy optimal value obtained } \\
\text { from modified interval-valued } \\
\text { fully fuzzy linear programming } \\
\text { problem }\end{array}$ \\
\hline Case 1 & $\begin{array}{l}{[(3.7756,4.2518,4.7833 ; .9),} \\
(3.8089,4.2518,4.9605 ; 1)]\end{array}$ & $\begin{array}{l}{[(3.947,4.2905,4.8053 ; .9),} \\
(3.8614,4.2905,4.9769 ; 1)]\end{array}$ \\
\hline Case 2 & $\begin{array}{l}(3.7756,4.2518,4.7833 ; .9) \\
(3.8089,4.2518,4.9605 ; 1)]\end{array}$ & $\begin{array}{l}{[(4.2570,4.6440,5.2245 ; .9),} \\
(4.1602,4.6440,5.4180 ; 1)]\end{array}$ \\
\hline Case 3 & $\begin{array}{l}(3.7756,4.2518,4.7833 ; .9), \\
(3.8089,4.2518,4.9605 ; 1)]\end{array}$ & $\begin{array}{l}{[(3.7756,4.2518,4.7833 ; .9),} \\
(3.8089,4.2518,4.9605 ; 1)]\end{array}$ \\
\hline Case 4 & $\begin{array}{l}(3.7756,4.2518,4.7833 ; .9), \\
(3.8089,4.2518,4.9605 ; 1)]\end{array}$ & $\begin{array}{l}{[(3.4478,3.7612,4.2314 ; .9),} \\
(3.3694,3.7612,4.3881 ; 1)]\end{array}$ \\
\hline Case 5 & $\begin{array}{l}{[(3.7756,4.2518,4.7833 ; .9),} \\
(3.8089,4.2518,4.9605 ; 1)]\end{array}$ & $\begin{array}{l}{[(3.7756,4.2518,4.7833 ; .9),} \\
(3.8089,4.2518,4.9605 ; 1)]\end{array}$ \\
\hline
\end{tabular}

Table 2. Results of original and modified interval-valued fully fuzzy linear programming problem 
cost coefficients corresponding to the fuzzy decision variables and the overall level of satisfaction of the decision maker about the statement that the optimal value will be units lies in.

c) For the original interval-valued fully fuzzy linear programming problem, the overall level of satisfaction of the decision maker for the remaining values of optimal value can be obtained as follows: Let represent the optimal value then the least value and greatest value of overall level of satisfaction of the decision maker for i.e. and can be obtained as follows:

$$
\begin{gathered}
\mu_{\tilde{A}^{L}}(x)=\left\{\begin{array}{cc}
\frac{.9(x-3.7756)}{(4.2518-3.7756)}, & 3.7756<x \leq 4.2518 \\
\frac{.9(4.7833-x)}{(4.7833-4.2518)}, & 4.2518 \leq x<4.7833 \\
0 \quad, & \text { otherwise }
\end{array}\right. \\
\mu_{\tilde{A}^{U}}(x)=\left\{\begin{array}{cl}
\frac{(x-3.8089)}{(4.2518-3.8089)}, & 3.8089<x \leq 4.2518 \\
\frac{(4.9605-x)}{(4.9605-4.2518)}, & 4.2518 \leq x<4.9605 \\
0 \quad, & \text { otherwise }
\end{array}\right.
\end{gathered}
$$

For the interval-valued fully fuzzy linear programming problem, obtained by changing the cost coefficients corresponding to the fuzzy decision variables $\tilde{x}_{1}$ and $\tilde{x}_{2}$ the overall level of satisfaction of the decision maker for the remaining values of optimal value can be obtained as follows:

Let ${ }^{X}$ represent the optimal value then the least value and greatest value of overall level of satisfaction of the decision maker for $x$ i.e. $\mu_{\tilde{A}^{L}}(x) \times 100$ and $\mu_{\tilde{A}^{U}}(x) \times 100$ can be obtained as follows:

$$
\mu_{\tilde{A}^{L}}(x)= \begin{cases}\frac{.9(x-3.947)}{(4.2905-3.947)}, & 3.947<x \leq 4.2905 \\ \frac{.9(4.8053-x)}{(4.8053-4.2905)}, & 4.2905 \leq x<4.8053 \\ 0 \quad, & \text { otherwise }\end{cases}
$$

$$
\mu_{\tilde{A}^{U}}(x)= \begin{cases}\frac{(x-3.8614)}{(4.2905-3.8614)}, & 3.8614<x \leq 4.2905 \\ \frac{(4.9769-x)}{(4.9769-4.2905)}, & 4.2905 \leq x<4.9769 \\ 0 \quad, & \text { otherwise }\end{cases}
$$

\section{Conclusions}

Till now, in previous works, there is no method that deals with the sensitivity analysis of such linear programming problems in which all the parameters are represented by interval-valued fuzzy numbers. In this paper, a new method is proposed for the sensitivity analysis. To illustrate the proposed method an interval-valued fully fuzzy sensitivity analysis problem, which cannot be solved by using any of the existing methods, is solved by using the proposed method. We emphasize that since there is no existing rule for the product of two arbitrary interval-valued $L R$ flat fuzzy numbers and its ranking function. This is an interesting research work for the future. 


\section{Acknowledgements}

The authors wish to thank to the editor and all the anonymous referees for the various suggestion which have led to an improvement in both, the quality and clarity of the paper. I, Dr. Amit Kumar, want to acknowledge the adolescent inner blessings of Mehar. I believe that Mehar is an angel for me and without Mehar's blessings it would not be possible to develop the idea proposed in this paper. Mehar is the lovely daughter of Parmpreet Kaur (Research Scholar under my supervision).

\section{References}

[1] L. A. Zadeh, "Fuzzy sets," Information and Control, vol. 8, pp. 338-353, 1965.

[2] H. Tanaka, T. Okuda and K. Asai, "On fuzzy mathematical programming," Journal of Cybernetics, vol. 3, pp. 37-46, 1974.

[3] R. E. Bellman and L. A. Zadeh, "Decision making in a fuzzy environment," Management Science, Vol. 17, pp. 141-164, 1970.

[4] H. J. Zimmermann, "Fuzzy programming and linear programming with several objective functions," Fuzzy Sets and Systems, vol. 1, pp. 45-55, 1978.

[5] H. R. Maleki, M. Tata and M. Mashinchi, "Linear programming with fuzzy variables," Fuzzy Sets and Systems, vol. 109, pp. 21-33, 2000.

[6] J. Chiang, "Fuzzy linear programming based on statistical confidence interval and interval-valued fuzzy set," European Journal of Operational Research, Vol. 129, pp. 65-86, 2001.

[7] N. Mahdavi-Amiri and S.H. Nasseri "Duality in fuzzy number linear programming by use of a certain linear ranking function," Applied Mathematics and Computation, vol. 180, pp. 206-216, 2006.

[8] K. Ganesan, and P. Veeramani, "Fuzzy linear programming with trapezoidal fuzzy numbers," Annals of Operations Research, Vol. 143, pp. 305-315, 2006.

[9] J. S. Su, "Fuzzy programming based on intervalvalued fuzzy numbers and ranking," International Journal Contemporary Mathematical Sciences, vol. 2, pp. 393-410, 2007.

[10] N. Mahdavi-Amiri and S.H. Nasseri, "Duality results and a dual simplex method for linear programming problems with trapezoidal fuzzy variables," Fuzzy Sets and Systems, vol 158, pp. 1961-1978, 2007.
[11] A. Ebrahimnejad, and S.H. Nasseri, "Using complementary slackness property to solve linear programming with fuzzy parameters," Fuzzy Information and Engineering, Vol. 3, pp. 233-245, 2009.

[12] F. Hosseinzadeh Lotfi, T. Allahviranloo, M. Alimardani Jondabeh and L. Alizadeh, "Solving a full fuzzy linear programming using lexicography method and fuzzy approximate solution," Applied Mathematical Modelling, Vol. 33, pp. 3151-3156, 2009.

[13] Ebrahimnejad, A. and Nasseri, S.H. (2010) 'A dual simplex method for bounded linear programmes with fuzzy numbers', International Journal of Mathematics in Operational Research, Vol. 2, pp.762-779.

[14] A. Ebrahimnejad, S. H. Nasseri and F. Hosseinzadeh Lotfi, "Bounded linear programs with trapezoidal fuzzy numbers," International Journal of Uncertainty, Fuzziness and Knowledge-Based Systems, Vol. 18, pp. 269-286, 2010.

[15] A. Ebrahimnejad, S. H. Nasseri, F. Hosseinzadeh Lotfi, and M. Soltanifar, "A primal-dual method for linear programming problems with fuzzy variables," European Journal of Industrial Engineering, Vol. 4, pp.189-209, 2010.

[16] S. H. Nasseri and A. Ebrahimnejad, "A fuzzy primal simplex algorithm and its application for solving flexible linear programming problems," European Journal of Industrial Engineering, vol. 4, pp. 372-389, 2010.

[17] B. Kheirfam and F. Hasani, "Sensitivity analysis for fuzzy linear programming problems with fuzzy variables," Advanced Modeling and Optimization, vol. 12, pp. 257-272, 2010.

[18] A. Kumar and N. Bhatia, "Sensitivity Analysis for fuzzy linear programming problems," RSFDGrC, Springer-Verlag Berlin Heidelberg, LNAI 6743, pp. 103-110, 2011.

[19] A. Kumar and N. Bhatia, "A New Method for Solving Fuzzy Sensitivity Analysis Problems," International Journal of Applied Science and Engineering, vol. 9, pp. 49-64, 2011.

[20] A. Ebrahimnejad, "Sensitivity analysis in fuzzy number linear programming problems," Mathematical and Computer Modeling, Vol. 53, pp.1878-1888, 2011.

[21] A. Kumar and N. Bhatia, "A New Method for Sensitivity Analysis of Fuzzy Linear Programming Problems," The Australian Society for Operations Research, vol. 31, pp. 2-12, N. 2012. 
[22] S. H. Nasseri and A. Ebrahimnejad, "Sensitivity analysis on linear programming problems with trapezoidal fuzzy variables," International Journal of Operation Research and Information Systems, vol. 2, pp. 22-39, 2011.

[23] N. Bhatia and A. Kumar, "Mehar's method for solving fuzzy sensitivity analysis problems with LR flat fuzzy numbers," Applied Mathematical Modelling, DOI: 10.1016/j.apm.2011.11.038, 2011.

[24] A. Hatami-Marbini and M. Tavana, "An extension of the linear programming method with fuzzy parameters," International Journal of Mathematics in Operational Research, vol. 3, pp. 44-55, 2011.

[25] P. Gupta and M. K. Mehlawat, "An algorithm for a fuzzy transportation problem to select a new type of coal for a steel manufacturing unit," TOP, vol. 15, pp. 114137, 2007.

[26] B. Gorzafczany "Approximate inference with interval-valued fuzzy sets-an outline." In: Proceedings of the Polish symposium on interval and fuzzy mathematics, Poznan, Poland, pp. 89-95, 1983. 